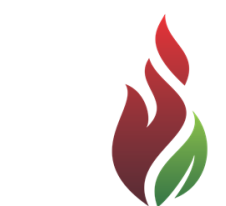

SUSTENERE

Publishing Corporation

\section{RISCO E TRANSPARÊNCIA NO BRASIL: UM ESTUDO SOBRE O GERENCIAMENTO DE RESULTADOS}

\section{RESUMO}

Dentre os mecanismos de Governança Corporativa propostos pela Teoria de Agência, a transparência é aquela que proporciona a redução da assimetria de informações entre gestores e investidores. Este artigo buscou verificar qual a relação existente entre o nível de transparência e o risco das empresas não-financeiras negociadas na BM\&FBovespa no período de 2003 a 2012. A dimensão transparência foi medida por meio das variáveis de gerenciamento de resultados (Earnings Management) propostas por Leuz et al. (2003) e o aspecto risco foi representado pelo Beta do CAPM (Capital Assets Pricing Model) e pelo Custo Médio Ponderado de Capital (WACC), conforme estudo de Lameira (2012). Como principais resultados, obteve-se uma relação significativa e positiva entre transparência e beta (proxy para o custo do capital próprio) e uma associação significativa e negativa entre transparência e WACC (proxy para o risco da estrutura interna de capital da empresa). Assim infere-se que uma maior qualidade da governança corporativa pode resultar no aumento do custo do capital próprio e, em contrapartida, na redução do custo de capital de terceiros, de tal forma que o custo médio ponderado de capital da firma seja reduzido.

PALAVRAS-CHAVES: Governança Corporativa; Transparência; Risco; Gerenciamento de Resultados.

\section{RISK AND DISCLOSURE IN BRAZIL: A STUDY ABOUT EARNINGS MANAGEMENT}

\section{ABSTRACT}

Among the Corporate Governance mechanisms proposed by the Agency Theory, disclosure is one that pursuits reduce the information asymmetry between managers and investors, contributing to increase the assertiveness of the investment allocation process. This article attempts to ascertain what is the relationship between the level of disclosure and the risk of non-financial companies listed on the BM\&FBovespa in the period of 2003-2012. Disclosure was measured using the variable of Earnings Management proposed by Leuz et al. (2003) and the risk aspect was represented by the Beta of the CAPM (Capital Assets Pricing Model) and the Weighted Average Cost of Capital (WACC) of the firm, according to a study of Lameira (2012). As main results, we obtained a significant and positive relationship between disclosure and beta (proxy for the cost of equity) and a significant and negative association between disclosure and WACC (proxy to measuring the risk of the capital structure of the company). Moreover, from this study, it appears that a higher quality of corporate governance can result in increased cost of capital and, in turn, reduce the cost of debt, so that the weighted average cost of capital the firm is reduced.

KEYWORDS: Corporate Governance; Disclosure; Risk; Earnings Management.
Revista Brasileira de

Administração Científica,

Aquidabã, v.5, n.3, Jul, Ago, Set,

Out, Nov, Dez 2014.

ISSN 2179-684X

SECTION: Articles

TOPIC: Finanças e Contabilidade

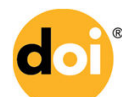

DOI: 10.6008/SPC2179-684X.2014.003.0012

Leandro de Carvalho Alves

Universidade Federal de Uberlândia, Brasil http://lattes.cnpq.br/3689670781585366 Ica@leandroalves.adm.br

Fabiolla Valeria Gonçalves Universidade Federal de Uberlândia, Brasil http://lattes.cnpq.br/8691295135798904 fabiolla.vg@hotmail.com

Fernanda Maciel Peixoto Universidade Federal de Minas Gerais, Brasil http://lattes.cnpq.br/5814414461245366 fmacielpeixoto@gmail.com

Received: 09/07/2014

Approved: 02/09/2015

Reviewed anonymously in the process of blind peer.

\section{Referencing this:}

ALVES, L. C.; GONÇALVES, F. V.; PEIXOTO, F. M.. A percepção de oportunidades no contexto do empreendedorismo social. Revista Brasileira de Administração Científica, Aquidabã, v.5, n.3, p.203221, 2014. DOI: http://dx.doi.org/10.6008/SPC2179 $684 X .2014 .003 .0012$ 


\section{INTRODUÇÃO}

Dentre os mecanismos de Governança Corporativa, Wong (2009) cita que a transparência é o mais valorizado pelas organizações. As estruturas de governança corporativa, sob o enfoque da transparência, têm como propósito: 1 - garantir aos acionistas minoritários o recebimento de informações confiáveis sobre o valor da empresa; 2 - garantir que os gestores e os grandes acionistas não irão enganar os acionistas minoritários quanto ao valor de seus investimentos e 3 garantir que os gestores e os acionistas majoritários não farão mau uso do poder conferido a eles em benefício próprio (BUSHMAN \& SMITH, 2003).

A análise das práticas de governança corporativa aplicada ao mercado de capitais envolve a transparência ou disclosure, a prestação de contas e a equidade de tratamento dos acionistas. Nesse sentido, para Castro Junior et al. (2011), a transparência é um aspecto importante para uma avaliação acertada das oportunidades de investimento no mercado financeiro, fomentando informações melhores e mais precisas para os acionistas e potenciais investidores, reduzindo assim a assimetria informacional e, portanto, o custo de capital próprio da empresa.

Mokhtar e Mellett (2013) citam que os riscos da falência pressionam as empresas por um maior disclosure. Os autores ainda ressaltam que os países desenvolvidos apresentam, no geral, um maior nível de divulgação voluntária de informações do que as empresas de países em desenvolvimento.

Neste contexto, esse trabalho se propõe a verificar qual a relação existente entre o nível de transparência e o risco das empresas não-financeiras negociadas na BM\&FBovespa entre os anos de 2002 a 2012. A hipótese principal deste estudo é de que quanto maior o grau de transparência menor será o risco da empresa. Para a mensuração da transparência utilizou-se como proxies as variáveis de gerenciamento de resultados propostas por Leuz et al. (2003). Já para a mensuração do risco, foram utilizadas as variáveis beta, em conformidade com o Modelo de Precificação de Ativos (CAPM - Capital Asset Pricing Model) e o Custo Médio Ponderado de Capital (WACC Weighted Average Cost of Capital), conforme sugerido por Lameira (2007) e Skaife et al. (2004). Para verificar uma possível associação entre as variáveis de interesse foi utilizado o modelo de regressão com dados em painel, visando lidar com a heterogeneidade das unidades cross-section.

Além dessa introdução, esse artigo contém uma segunda seção na qual foi desenvolvido o referencial teórico que aborda os principais conceitos de governança corporativa, risco e a relação existente entre eles. Na terceira seção apresentou-se a metodologia de pesquisa que guiou a análise de resultado apresentada na quarta seção, permitindo verificar a possível influência da transparência no risco das empresas não financeiras. A quarta seção engloba a análise dos resultados e a quinta e última seção as conclusões proporcionadas por essa pesquisa. 


\section{REVISÃO TEÓRICA}

\section{Governança Corporativa}

Sabe-se que a Teoria da Agência e os conceitos de Governança Corporativa (GC) são indissociáveis. Um dos estudos precursores sobre GC no contexto norte-americano foi o de Berle e Means (1932), que identificaram a origem do conflito de agência como sendo a separação entre a propriedade e o controle do capital.

Diante dos conflitos e custos de agência e da assimetria de diretos entre insiders e outsiders, a adoção de mecanismos de GC é essencial para o desenvolvimento corporativo. Para Wong (2009), os mecanismos de GC adotados consistem em: 1 - Melhorar a transparência através de uma maior divulgação informacional; 2 - Conselho de administração, que ocupa uma posição estratégica no governança corporativa; 3 - Alinhamento de interesses dos agentes através de remuneração baseada em desempenho e outros incentivos financeiros; 4 - Reforçar os direitos dos acionistas e 5 - Responsabilização financeira dos gestores, auditores externos, bancos de investimento e outros intermediários, para garantir a diligência, lealdade e honestidade (WONG, 2009).

Tomando-se a transparência como um dos objetos de estudo, no âmbito nacional o Instituto Brasileiro de Governança Corporativa (IBCG, 2010) define que transparência é o desejo de conceder para as partes interessadas informações que sejam de seu interesse e não apenas as que são impostas por disposições de regulamentos e leis. Sobre essas possibilidades de divulgação das informações, Lanzana (2004) diz que as empresas podem fornecer informações através de "seus relatórios financeiros, incluindo demonstrativos financeiros, notas de rodapé, análise e discussão por parte dos gestores, assim como outros documentos exigidos pelos órgãos reguladores". Esse estudo corrobora com o raciocínio de que a informação contábil reduz a assimetria e minimiza o risco dos investidores, possibilitando o aumento dos fundos para o mercado de capitais e, consequentemente, reduzindo o risco de liquidez.

Castro Junior et al. (2011) adicionam que para incentivar um maior nível de transparência nas companhias, após a implantação da lei Sarbanes-Oxley (SOX) em 2002 nos Estados Unidos, outros países fortaleceram a legislação do mercado de capitais através dos órgãos reguladores. $O$ mesmo ocorreu em 2000 no Brasil, com a instituição, pela BM\&F Bovespa, dos Níveis Diferenciados de Governança Corporativa (NDGC). Ainda com relação à SOX, Andrade e Rossetti (2006) complementam que além da transparência (disclosure), os benefícios dessa legislação podem ser condensados em três grandes grupos: o compliance (conformidade legal), a accountability (prestação de contas) e o fairness (senso de justiça).

Ainda no quesito legal, La Porta (1998) afirmam que tanto a qualidade do enforcement (capacidade ou poder de aplicação da lei) quanto o próprio conteúdo da lei são elementos importantes da Governança Corporativa. Nesse sentido, mesmo que exista um enforcement legal, 
Bueno (1999) confirma que a divulgação das informações, não precisa ser necessariamente legal ou formal, podendo ocorrer de forma voluntária ou informal; "o importante é que as informações sejam as mesmas para todos, evitando a práticas de informações privilegiadas (insider information)".

Partindo para a transparência contábil-financeira, Bushman e Smith (2003) definem que a mesma permite a avaliação das oportunidades de investimento em uma economia por parte dos gestores na escolha de projetos, e por parte dos investidores na alocação de recursos. Sob ótica similar, Skinner (1993) expõe que os gestores recebem incentivos para elevar o nível de disclosure quando as ações da empresa estão sobrevalorizadas, divulgando notícias ruins para evitar problemas legais. Por outro lado, quando as notícias divulgadas são boas e as ações estão subavaliadas, os gestores então aumentariam a qualidade da informação fornecida ao mercado, para aumentar a credibilidade da empresa resolvendo o problema da subavaliação.

\section{Transparência e Risco}

A transparência como um dos instrumentos de Governança Corporativa é, para Wong (2009), um dos mecanismos mais disseminados nas empresas e tem por objetivos: (1) prover informações de desempenho e risco; (2) garantir uma padronização das informações; (3) reduzir o conflito de agência entre insiders e outsiders; (4) gerenciar conflitos de interesses entre proprietários, gestores, intermediários e demais stakeholders.

Sobre essa temática, Lanzana, Silveira e Famá (2006) verificaram se a relação entre GC e disclosure é complementar ou substituta, em 161 companhias abertas da Bovespa, no período de 2002. Se complementar, as empresas com melhores práticas de governança apresentariam maior disclosure. E se a relação fosse substituta as empresas com piores práticas de governança apresentariam um maior disclosure. Os resultados não assinalaram uma única hipótese (complementação ou substituição) para as dimensões de governança. As variáveis relacionadas à estrutura de propriedade apontaram uma relação de substituição entre governança e disclosure, enquanto que as variáveis relativas ao conselho indicaram uma relação de complementação.

Numa linha semelhante Malacrida e Yamamoto (2006) tratando de disclosure, estudaram a relação entre o nível de evidenciação dos dados contábeis e a volatilidade do retorno das ações de 42 empresas do lbovespa em 2002. Os resultados apontaram que maior nível médio de disclosure implica em menor volatilidade média do retorno das ações, o que indica que os investidores estariam dispostos a pagar um maior preço por ações de empresas transparentes.

Abordando a transparência, qualidade e acurácia das informações divulgadas, Fernandes e Ferreira (2008) investigaram a evolução do gerenciamento de resultados e valor da firma em 24.000 empresas em 43 países entre 1990 e 2003. Os autores verificaram que oportunidades de investimento, dependência de financiamento externo, dispersão de propriedade, manutenção de 
caixa, ambiente de forte proteção legal ao investidor e maior visibilidade e acesso aos mercados de capitais globais tendem a diminuir o gerenciamento de lucros.

Na mesma linha Lopes e Walker (2008) analisaram a transparência dos relatórios contábeis no Brasil sob a ótica da firma, entre 1998 e 2004. A justificativa para tal estudo foi de que o Brasil apresenta uma estrutura insatisfatória de governança, enforcement deficiente, fraca proteção legal aos acionistas, incentivos à manipulação de lucros, mercado financeiro instável e volátil e com importantes oportunidades de crescimento. Os resultados apuraram que padrões mais exigentes de governança e a decisão de emitir ADR's estão negativamente relacionados à manipulação de relatórios contábeis em organizações do mesmo ambiente institucional.

No estudo de Leuz et al. (2003) são quatro as variáveis de gerenciamento de resultados: (1) a suavização dos lucros operacionais; (2) a correlação entre accruals e o fluxo de caixa das operações; (3) a magnitude dos accruals; (4) a pontuação agregada do gerenciamento de resultados (média aritmética da pontuação em cada uma das outras três variáveis). Dessa forma, com mais de 8.000 empresas de 31 países durante o período de 1990 e 1999 e utilizando o mínimo quadrado em dois estágios, os autores concluem que quanto maior o nível de transparência e o enforcement legal de um mercado por consequência os minoritários estarão mais protegidos e, por isso, existe menos manipulação de resultados por parte dos gestores e dos majoritários. Já nas pesquisas brasileiras, Martinez (2013) destaca que as métricas de detecção de gerenciamento de resultados podem ser agrupadas em: (1) accruals discricionários; (2) gerenciamento por decisões operacionais; (3) distribuição de lucros; (4) contas específicas - specific accruals; (5) indicadores de income smoothing.

Espera-se que quanto mais transparentes forem as informações prestadas por um empresa, menor será o risco assumido ao se investir nessa firma. Correia (2008) prova estatisticamente essa relação através de regressões entre um índice de governança proposto e validado por ela e a taxa de retorno ajustada ao risco. Em seu trabalho, Correia (2008) elabora um índice de governança (IQG) que, através da análise de componentes principais, mede a qualidade dos cinco mecanismos de governança corporativa para empresas brasileiras. Nesse índice a transparência representa, sozinha, cerca de $39 \%$ do total do indicador. No estudo de Peixoto (2012), que apresenta a evolução dos mecanismos de CG entre os anos de 2000 e 2009, a variável transparência foi pouco representativa na composição média do índice de governança. No período de maior representatividade, 2002, a transparência contribuiu com $29,66 \%$ do índice. Contudo, ao longo do período essa representatividade foi reduzida chegando a 9,54\% no ano de 2007.

Srour (2005) objetivando verificar a eficácia dos mecanismos de contratos privados como provedores de proteção aos investidores externos no Brasil, em empresas de capital aberto no período entre 1997 e 2001, concluíram que as empresas podem se envolver com a proteção aos acionistas por meio do lançamento de ADR Nível II ou com a presença do Novo Mercado, e em períodos de crise tais firmas tendem a apresentar maiores retornos e distribuir mais lucros em forma de dividendos. 
Mokhtar e Mellett (2013) buscaram medir o grau de informação de risco obrigatório e voluntário, e investigar o impacto da concorrência, governança corporativa e estrutura de propriedade sobre os relatórios de risco prático em relatórios anuais de 105 empresas egípcias listadas em 2007. Os resultados evidenciaram um baixo nível de cumprimento das exigências de relatórios de risco obrigatório, é indicada uma baixa extensão de denúncia espontânea do risco, com uma tendência retrógrada se comparada à divulgação qualitativa de risco para o futuro.

De outra forma, Lameira (2007) correlaciona a governança corporativa - medida através de um questionário de 20 perguntas binárias sobre os cinco instrumentos de governança - com o risco através das variáveis beta local, beta internacional, volatilidade do retorno das ações, risco não sistemático, custo médio ponderado de capital e a diferença entre o retorno da ação e o retorno da renda fixa. Como resultado, Lameira (2007) encontrou uma relação negativa e significativa entre governança corporativa e risco. Nesse mesmo sentido, Skaife et al. (2004), também encontraram uma relação negativa entre a transparência e o risco medido através do beta. Utilizando uma amostra de cerca de 1.500 grandes empresas no período da década de 1990, os autores concluíram que o Índice de Governança Corporativa desenvolvido por eles explica cerca de $14 \%$ da variação do beta. Skaife et al. (2004) averiguaram que o beta é uma variável de controle adequada para os modelos cuja variável dependente é o custo implícito de capital. Para mensurar a variável risco, os autores utilizaram como proxy, o custo de capital (WACC) em $t+1$. Assim, os autores encontraram uma relação negativa e estatisticamente significativa entre a governança corporativa e o risco em um modelo controlado pelo próprio beta, o tamanho da empresa e o valor market-to-book.

Por outro lado, em uma pesquisa que buscava relacionar governança corporativa com o custo de capital próprio de empresas brasileiras, Salmasi (2007) encontrou uma relação positiva e significativa entre a adesão aos níveis diferenciados de governança corporativa da BM\&FBovespa e o custo de capital próprio quando se utilizou o beta $(\beta)$ do CAPM. Contudo, ao se utilizar como proxy do custo de capital próprio o modelo de três fatores de Fama e French os resultados obtidos foram não significativos. Apesar de no estudo de Salmasi (2007) o beta do CAPM ter sido utilizado como proxy do custo do capital próprio, essa variável também serve como proxy do risco sistemático da empresa, conforme trabalhos de Skaife et al. (2004), Lameira (2007) e Peixoto (2012).

\section{METODOLOGIA}

\section{Classificação da Pesquisa, População e Amostra}

Quanto aos objetivos de pesquisa esse estudo se classifica como uma pesquisa quantitativa e descritiva, sendo a sua população composta por todas as empresas listadas na BM\&F BOVESPA que foram negociadas no período de 2003 a 2012, o que totaliza, considerando as informações do banco de dados da Economática ${ }^{\circledR}, 688$ empresas. A partir dessa população, foram excluídas as 
empresas do setor financeiro e de seguros, pois elas possuem critérios de análise distintos das demais empresas devido à seus níveis de endividamento e alavancagem específicos.

Um segundo filtro adotado no trabalho foi com relação à liquidez. Baseando-se no trabalho de Silveira et al. (2008), foram excluídas as empresas-ano que, de acordo com o índice de liquidez calculado pela Economática ${ }^{\circledR}$, apresentaram índice de liquidez anual inferior a $0,001 \%$ do índice correspondente à empresa com ações mais líquidas no período do estudo.

Quadro 1: Amostra final

\begin{tabular}{|c|c|}
\hline Ano & Empresas-ano \\
\hline 2003 & 107 \\
\hline 2004 & 118 \\
\hline 2005 & 122 \\
\hline 2006 & 145 \\
\hline 2007 & 202 \\
\hline 2008 & 200 \\
\hline 2009 & 202 \\
\hline 2010 & 209 \\
\hline 2011 & 211 \\
\hline 2012 & 212 \\
\hline Total Empresas-ano & $\mathbf{1 . 7 2 8}$ \\
\hline Total de Empresas & $\mathbf{2 7 2}$ \\
\hline
\end{tabular}

Considerando essas restrições, a amostra final foi composta por 272 empresas, que representam, conforme demonstrado no Quadro 1, um total de 1.728 empresas-ano. Assim, com o intuito de evitar o viés de sobrevivência, com a amostra final formou-se um painel não balanceado que serviu de base para as estimativas econométricas desse trabalho.

\section{Definição das Variáveis}

A definição das variáveis da pesquisa foi embasada na literatura nacional e internacional sobre Governança Corporativa. Essas variáveis são indicadores de qualidade da Governança Corporativa das empresas, mais especificamente sobre o mecanismo de transparência, indicadores contábeis e medidas de risco.

\section{Variáveis Dependentes}

Levando em consideração as pesquisas de Skaife et al. (2004), Lameira (2007) e Peixoto (2012), foram selecionadas as seguintes variáveis dependentes para a estimação dos modelos:

a) Beta (B): representa o risco sistemático da ação e foi calculado através da regressão do prêmio de risco mensal do título contra o prêmio de risco mensal do IBOVESPA (indicador de mercado) nos sessenta meses anteriores ao período de análise, de acordo com o modelo CAPM (Capital Asset Pricing Model). Considerou-se a taxa Selic como proxy do ativo livre de risco, conforme os trabalhos de Barros et al. (2003) e Rogers e Securato (2009).

$$
P R_{i t}=\beta_{i t}+P R_{M t}
$$


Onde: $P R_{i t}=$ Prêmio de risco (retorno do título - retorno do ativo livre de risco) do título i no tempo $t ; \beta_{i t}=$ Risco sistemática da ação; $P R_{M t}=$ Prêmio de risco (retorno do mercado - retorno do ativo livre de risco) do mercado (IBOVESPA) no tempo $t$.

b) Custo médio ponderado de capital - CMPC (WACC): é uma proxy do custo de capital da empresa e representa o risco da estrutura de capital da mesma. Esse custo pode ser calculado, de acordo com Damodaran (2002), conforme a seguinte formulação:

$$
W A C C=\left\{\frac{[E B I T x(1-T)+D-I]}{(E Q U I T Y+D E B I T)} x[1+(1-\text { payout }) x R O A]\right\}+[(1-\text { payout }) x R O A]
$$

Onde: WACC (Weighted Average Cost of Capital) = Custo médio ponderado de capital da empresa; EQUITY = Valor de mercado das ações da empresa; DEBT = Valor contábil da dívida da empresa; EBIT = Lucros antes de juros e impostos; $T$ = Alíquota de imposto de renda; $D=$ Valor da depreciação incorrida no período; $I$ = Valor do investimento feito pela empresa; Payout= Percentual do lucro destinado ao pagamento de dividendos; ROA = Razão entre o lucro operacional e os ativos totais da empresa.

Para facilitar a interpretação dos resultados, utilizou-se nesse estudo a razão entre o WACC (obtido pela equação 1) e o Ativo Total, constituindo o coeficiente WACC/AT. Além disso, como os efeitos da governança são observáveis no custo de capital somente no ano posterior, conforme argumentado por Skaife et al. (2004) e Lameira (2007), esse trabalho também adotou o indicador WACC/AT em $t+1$. Para o cálculo do WACC em $t+1$ todas as informações foram retiradas das demonstrações do segundo trimestre de cada ano. Por fim, seguindo Peixoto (2012) adotou-se também o indicador WACC/AT no tempo corrente, garantido, assim, a verificação de ambas as hipóteses de relação em $\mathrm{t}$ ou em $\mathrm{t}+1$.

\section{Variáveis Independentes}

Para a mensuração da transparência, utilizaram-se nesse estudo as variáveis propostas por Leuz et al. (2003). Esses autores utilizam medidas para gerenciamento de resultados (earnings management) como proxies para a qualidade das informações publicadas, ou seja, a transparência dos lucros contábeis. Essa mesma abordagem foi utilizada em outros estudos tais como: Fernandes e Ferreira (2007), Correia (2008) e Peixoto (2012).

Apesar de no estudo original Leuz et al. (2003) consolidar em um único indicador quatro formas de mensuração de gerenciamento de resultados, nesse estudo foi utilizado nas regressões as formas individuais de cada medida de manipulação dos resultados. Além disso, devido às características operacionais de cálculo (que implicam em uma quantidade mínima de observações), este estudo não utilizou a medida de prevenção de pequenas perdas (small loss avoidance). Com isso, utilizaram-se as seguintes variáveis:

a) Transparência 1 (T1): Mede a suavização dos lucros operacionais através da alteração dos accruals (contas não caixa). É mensurada pela relação entre o desvio padrão do lucro depois do imposto de renda e o desvio padrão do fluxo de caixa das operações, ambos controlados, 
conforme modelagem de Leuz et al. (2003), pelo ativo total do período anterior.

$$
T 1=\frac{\sigma\left(L D I R_{i t}\right)}{\sigma\left(F C O_{i t}\right)}
$$

Onde: T1= Transparência 1; LDIRit = Lucro depois do imposto de renda da empresa $i$ no tempo $t$; FCOit = Fluxo de caixa das operações da empresa $\mathrm{i}$ no tempo $t$.

Seguindo ainda a metodologia do estudo de Leuz et al. (2003), o cálculo de T1 foi operacionalizado utilizando dados da Economática ${ }^{\circledR}$ e as seguintes formulações:

$$
\begin{aligned}
& A C C_{i t}=\left(\Delta C A_{i t}-\triangle C A S H_{i t}\right)-\left(\Delta C L_{i t}-\Delta S T D_{i t}-\Delta T P_{i t}\right)-D e p_{i t} \\
& C F O_{i t}=L D I R_{i t}-A C C_{i t}
\end{aligned}
$$

Onde: $A C C_{i t}=$ Accruals, itens contábeis que não movimentam efetivamente o caixa; $\triangle C A_{i t}$ = Variação nos ativos circulantes totais; $\triangle C A S H_{i t}=$ Variação no disponível e investimentos de curto prazo; $\Delta C L_{i t}=$ Variação nos passivos circulantes totais; $\Delta S T D_{i t}=$ Variação na dívida de curto prazo incluída nos passivos circulantes; $\Delta T P_{i t}=$ Variação no imposto de renda a pagar; Dep $p_{i t}=$ Despesa de depreciação e amortização.

Ressalta-se que baixos valores de T1 sugerem o gerenciamento dos lucros por parte dos insiders.

b) Transparência 2 (T2): Mede a suavização e correlação entre a variação dos accruals e a variação nos fluxos de caixa das operações, ambos controlados pelo ativo total do período anterior.

$T 2=\rho(\triangle A C C ; \triangle F C O)$

Onde: $\mathrm{ACC}_{\mathrm{it}}=$ Variação dos accruals; $\Delta \mathrm{FCO}=$ Variação do fluxo de caixa da operação.

Para Leuz et al. (2003), grandes correlações (T2) indicam, ceteris paribus, que os resultados foram gerenciados.

c) Transparência 3 (T3): Mede a qualidade dos lucros reportados através da magnitude dos accruals.

$$
T 3=\frac{|A C C|}{|F C O|}
$$

Onde: $|\mathrm{ACC}|=$ Valor modular dos accruals; $|\mathrm{FCO}|=$ Valor modular do fluxo de caixa da operação.

Segundo a interpretação de Leuz et al. (2003), a magnitude dos accruals é uma proxy para o nível em que os insiders gerenciam os resultados.

d) Média das Transparências (TM): Assim como Leuz et al. (2003), também foi utilizada a variável média das transparências, que foi obtida através da média aritmética de T1, T2 e T3 para cada empresa em cada um dos anos do estudo.

\section{Variáveis de Controle}

O principal objetivo na utilização das variáveis de controle é a possível redução de vieses causados pela omissão de variáveis explicativas nos modelos propostos. Assim, seguindo a 
proposta de Gompers, Ishii e Metrick (2003), Silveira (2004), Lameira (2007), Correia (2008), Liu, Uchida e Yang (2012) e Peixoto (2012) este estudo utilizou-se das seguintes variáveis de controle para governança corporativa:

a) Volatilidade da ação (VOL): mede a volatilidade anual de cada ação. Esta variável foi extraída do Economática ${ }^{\circledR}$, normalizada para as regressões através da raiz quadrada e usada somente quando a variável dependente foi o beta.

b) Beta (B): representa o risco sistemático da ação e foi calculado através da regressão do prêmio de risco mensal do título contra o prêmio de risco mensal do IBOVESPA (indicador de mercado) nos sessenta meses anteriores ao período de análise, de acordo com o modelo CAPM (Capital Asset Pricing Model). Considerou-se a taxa Selic como proxy do ativo livre de risco, conforme os trabalhos de Barros et al. (2003) e Rogers e Securato (2009).

c) Índice book-to-market (BTM): representa a sub ou sobre valorização do ativo e foi calculado como o logaritmo natural da razão entre valor patrimonial por ação e o seu preço.

$$
B T M=\ln \left(\frac{P L / Q T D}{C O T}\right)
$$

Onde: BTM = Índice book-to-market; PL = Patrimônio líquido da empresa; QTD = Quantidade de ações; COT = Preço de fechamento da ação.

d) Valor de mercado (VM): representa uma proxy para tamanho da empresa e foi calculado como o logaritmo natural do indicador de valor de mercado retirado da Economática ${ }^{\circledR}$.

e) Liquidez em bolsa (LIQ): indicador extraído da Economática ${ }^{\circledR}$ que representa o volume relativo de negociações com a ação e pode ser calculado conforme se segue:

$$
L I Q=100 x\left(\frac{p}{P}\right) \times \sqrt{\left(\frac{n}{N}\right) \times\left(\frac{v}{V}\right)}
$$

Onde: LIQ = Liquidez em bolsa; $p$ = número de dias em que houve pelo menos um negócio com a ação dentro do período escolhido; $\mathrm{P}$ = número total de dias do período escolhido; $\mathrm{n}$ = número de negócio com a ação dentro do período escolhido; $\mathrm{N}=$ número de negócios com todas as ações negociadas na bolsa dentro do período escolhido; $v$ = volume em dinheiro com a ação dentro do período escolhido; $\mathrm{V}$ = volume em dinheiro com todas as ações negociadas na bolsa dentro do período escolhido.

f) Adesão aos níveis diferenciados de governança corporativa da BM\&FBOVESPA (NDGC): indica a classificação das empresas nos níveis diferenciados de governança corporativa (Nível 1, Nível 2 ou Nível 3 - Novo Mercado). Para as regressões foram criadas uma variável dummy para cada nível de adesão.

Quadro 2: Definição das Variáveis

\begin{tabular}{|c|l|c|l|}
\cline { 2 - 4 } \multicolumn{1}{c|}{} & \multicolumn{1}{c|}{ Nome } & Variável & \multicolumn{1}{c|}{ Definição } \\
\hline & & B & $\begin{array}{l}\text { Risco sistemático da ação e foi calculado através da regressão do prêmio } \\
\text { de risco mensal do título contra o prêmio de risco mensal do IBOVESPA } \\
\text { (indicador de mercado) nos sessenta meses anteriores ao período de } \\
\text { análise. }\end{array}$ \\
\hline
\end{tabular}




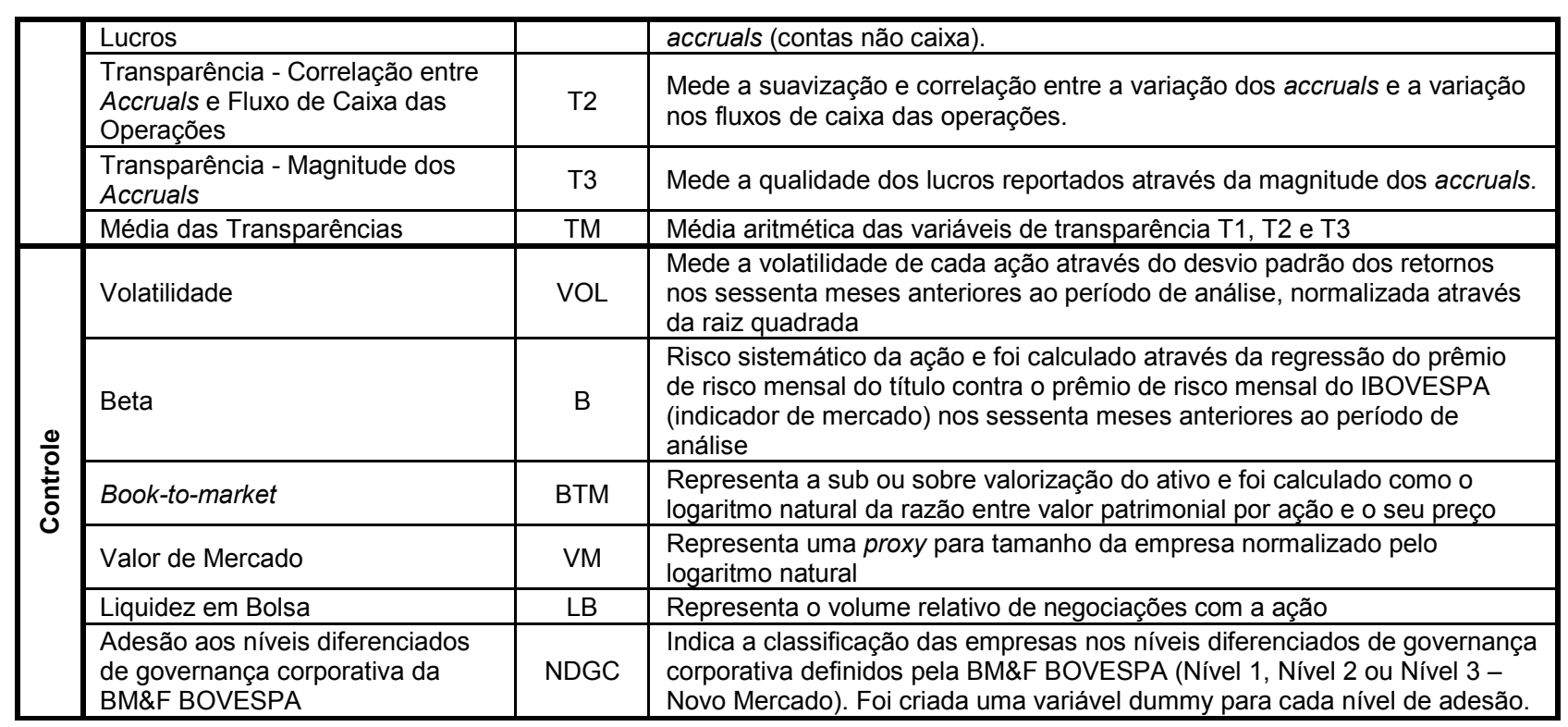

Além das variáveis citadas, todos os modelos também foram controlados por dummies para o Setor da Economática e Ano. Dessa forma, o Quadro 2 apresenta o resumo das variáveis utilizadas nesse estudo.

\section{Definição dos Modelos Econométricos}

A estimativa com dados em painéis deve começar, de acordo com Greene (2008), com o teste de Lagrange Multiplier (LM) que tem como hipótese nula que os interceptos são iguais. Dessa forma, se $\mathrm{H}_{0}$ não for rejeitada, deve-se utilizar o modelo Pooled. Por outro lado, caso $\mathrm{H}_{0}$ seja rejeitada deve-se optar pelos modelos de efeitos fixos ou aleatórios.

A definição entre a utilização de modelos de efeitos fixos ou aleatórios pode ser realizada através do teste proposto por Hausman (1978). Esse teste tem a hipótese nula de que os efeitos dos interceptos são correlacionados com os $x_{i t}$, situação em que se deve eleger o modelo de efeitos fixos. Ao contrário, rejeitando-se $\mathrm{H}_{0}$ o modelo a ser utilizado será o de efeitos aleatórios.

Nessa pesquisa, após a aplicação dos testes propostos por Breusch e Pagan (1980) e Hausman (1978), optou-se pelo método de regressão de Efeitos Aleatórios aplicado a um painel desbalanceado para todos os modelos analisados, exceto para o modelo com WACC $\mathrm{t}-1$ com TM.

Além disso, como os dados em painéis podem apresentar tantos os problemas encontrados nos dados em cortes transversais quanto nas séries temporais, os modelos também foram testados contra as premissas de homocedasticidade e ausência de autocorrelação dos termos de erros. Para isso, utilizaram-se, respectivamente, os testes de Baum (2001) e de Wooldridge (2002) que resultaram na não rejeição das hipóteses nulas de homocedasticidade e ausência de autocorrelação em todos os casos. Assim, seguindo as orientações de Hoechle (2007), todas as estimativas foram obtidas com os erros padrão robustos aos distúrbios de heterocedasticidade e autocorrelação através da opção vce(cluster id) no software Stata $13^{\circledR}$. 
Por fim, testou-se a endogeneidade dos modelos através do teste de Durbin-Wu-Hausman. Esse teste foi operacionalizado no Stata $13^{\circledR}$ com o comando xtivreg2 e obteve como resultado a indicação de que todos os modelos são exógenos.

Assim, os seguintes modelos econométricos foram utilizados para análise das regressões com dados em painéis:

$$
V D_{i t}=\beta_{1} V I_{i t}+\beta_{2} V C_{i t}+\left(\beta_{0 i}+\varepsilon_{i t}\right)
$$

Modelo de Efeitos Aleatórios onde: $\mathrm{VD}_{\mathrm{it}}=$ Variável dependente: Beta, WACC no período corrente ou WACC em $\mathrm{t}+1 ; \beta_{1}=$ Coeficiente da variável independente; $\mathrm{VI}_{\mathrm{it}}=$ Variável independente: $\mathrm{T} 1, \mathrm{~T} 2, \mathrm{~T} 3$ ou $\mathrm{TM} ; \beta_{\mathrm{n}}=$ Coeficiente de cada variável de controle; $\mathrm{VC}_{\mathrm{it}}=$ Variáveis de controle: Volatilidade, Beta (somente para WACC e WACC em t + 1), Book-to-market, Valor de Mercado, Liquidez em Bolsa, Dummies para a adesão aos níveis diferenciados de governança corporativa, Dummies para o setor da Economática, Dummies para os anos; $\left(\beta_{0 \mathrm{i}}+\varepsilon_{\mathrm{it}}\right)=$ Interceptos + Termos de erros.

$$
V D_{i t}=\beta_{0 i}+\beta_{1} V I_{i t}+\beta_{2} V C_{i t}+\varepsilon_{i t} \quad \text { [II] }
$$

Modelo de Efeitos Fixos onde: $\mathrm{VD}_{\mathrm{it}}=$ Variável dependente: WACC em $\mathrm{t}+1 ; \beta_{1}=$ Coeficiente da variável independente; $\mathrm{VI}_{\mathrm{it}}=$ Variável independente: $\mathrm{T} 3$ ou $\mathrm{TM} ; \beta_{\mathrm{n}}=$ Coeficiente de cada variável de controle; $\mathrm{VC}_{\mathrm{it}}=$ Variáveis de controle: Volatilidade, Beta, Book-to-market, Valor de Mercado, Liquidez em Bolsa, Dummies para a adesão aos níveis diferenciados de governança corporativa, Dummies para o setor da Economática, Dummies para os anos; $\left(\beta_{0 \mathrm{i}}+\varepsilon_{\mathrm{it}}\right)=$ Interceptos + Termos de erros.

\section{RESULTADOS E DISCUSSÃO}

Com o intuito de fornecer uma visão geral dos dados utilizados nessa pesquisa, a Tabela 1 apresenta as estatísticas descritivas das variáveis.

Com relação às medidas de risco, observa-se uma grande variabilidade entre as empresas independente da variável analisada. Essa dispersão pode ser explicada tanto pelas características das empresas que compõem a amostra - envolvendo praticamente todos os setores da economia e com empresas de diversos tamanhos - quanto pelo período escolhido que engloba subperíodos de pré-crise, crise e pós-crise.

Já as variáveis de transparência, que representam uma proxy da governança corporativa, podem ser agrupadas em dois segmentos de dispersão. Um primeiro grupo, que apresenta maior dispersão, contendo as variáveis T1 e TM que tem como médias 0,689 e 0,243 respectivamente. Já as variáveis T2 e T3 podem ser reunidas em um segundo grupo que apresenta baixa dispersão, tendo, essas variáveis, médias de 0,165 e -0,003 respectivamente. 
Tabela 1: Resumo das Estatísticas Descritivas

\begin{tabular}{lccccc}
\hline \multicolumn{1}{c}{ Variáveis } & Obs. & Média & Desvio Padrão & Mínimo & Máximo \\
\hline B & 1.707 & 0,895 & 1,017 & $-18,206$ & 9,028 \\
WACC & 1.587 & $-1,249$ & 1,764 & $-37,906$ & 6,079 \\
WACC ${ }^{+1}$ & 929 & $-1,386$ & 3,363 & $-90,568$ & 0,609 \\
T1 & 1.300 & 0,689 & 0,771 & 0,039 & 10,053 \\
T2 & 1.200 & 0,165 & 0,214 & 0,000 & 1,000 \\
T3 & 1.537 & $-0,004$ & 0,030 & $-1,000$ & 0,000 \\
TM & 1.555 & 0,243 & 0,302 & $-0,202$ & 3,676 \\
VOL & 1.043 & 52,573 & 27,346 & 2,700 & 234,600 \\
BTM & 1.541 & $-0,381$ & 1,008 & $-5,443$ & 3,357 \\
VM & 1.612 & 13,876 & 2,094 & 6,596 & 19,714 \\
LB & 1.728 & 0,161 & 0,408 & 0,000 & 4,265 \\
NDGC & 1.728 & 1,124 & 1,396 & 0,000 & 3,000 \\
\hline
\end{tabular}

No que se refere à matriz de correlação (Tabela 2), observa-se que as variáveis de transparência, exceto T3, possuem correlação negativa com a variável NDGC. Essas correlações negativas, ainda que baixas, contrariam o que se espera da literatura, pois de acordo com Leuz et al. (2003) as variáveis de transparência possuem sentido positivo com a governança corporativa. Essa divergência pode ser explicada pelo fato de a adesão aos Níveis Diferenciados de Governança Corporativa da BM\&FBovespa ser voluntária, o que pode gerar distorções nessa variável como proxy do real nível de governança das empresas.

No mesmo sentido, o tamanho da empresa, medido pelo valor de mercado (VM), também apresenta correlação negativa com as todas variáveis de transparência, indicando que quanto maior a empresa mais os seus resultados são gerenciados (pior é a governança corporativa). Em contrapartida, ao se analisar a correlação do tamanho da empresa com a variável NDGC encontrase uma correlação positiva, da mesma forma que no estudo de Salmasi (2007).

Ainda com relação à Tabela 2, as grandes correlações encontradas entre T1 e TM e entre T2 e TM não interferem nas análises, visto que essas variáveis não compõem o mesmo modelo.

Tabela 2: Matriz de correlação entre as variáveis

\begin{tabular}{|c|c|c|c|c|c|c|c|c|c|c|c|c|}
\hline & B & WACC & $\begin{array}{c}\text { WACC } \\
t+1\end{array}$ & T1 & T2 & T3 & TM & VOL & BTM & VM & LB & NDGC \\
\hline B & 1 & & & & & & & & & & & \\
\hline WACC & 0,053 & 1 & & & & & & & & & & \\
\hline $\begin{array}{l}\text { WACC } \\
t+1\end{array}$ & 0,044 & 0,719 & 1 & & & & & & & & & \\
\hline T1 & 0,023 & 0,000 & 0,020 & 1 & & & & & & & & \\
\hline T2 & $\overline{0}, \overline{042}$ & $-0,096$ & $-0,022$ & 0,548 & 1 & & & & & & & \\
\hline T3 & 0,019 & $-0,019$ & $-0,010$ & 0,007 & 0,010 & 1 & & & & & & \\
\hline TM & 0,029 & $-0,004$ & 0,009 & 0,970 & 0,711 & 0,037 & 1 & & & & & \\
\hline VOL & 0,200 & 0,070 & 0,107 & 0,088 & 0,114 & 0,035 & 0,029 & 1 & & & & \\
\hline BTM & $\begin{array}{c}- \\
0,004\end{array}$ & $-0,444$ & $-0,151$ & 0,006 & 0,169 & $\begin{array}{c}- \\
0,004\end{array}$ & 0,051 & $\begin{array}{c}- \\
0,042\end{array}$ & 1 & & & \\
\hline VM & 0,050 & 0,268 & 0,053 & $\begin{array}{c}- \\
0,030\end{array}$ & $\begin{array}{c}- \\
0,124\end{array}$ & $0, \overline{0}$ & $\begin{array}{c}- \\
0,018\end{array}$ & $\begin{array}{c}- \\
0,296\end{array}$ & $\begin{array}{c}- \\
0,401\end{array}$ & 1 & & \\
\hline LB & 0,054 & 0,110 & 0,064 & $\begin{array}{c}- \\
0,039\end{array}$ & $\begin{array}{c}- \\
0,056\end{array}$ & 0,009 & $\begin{array}{c}- \\
0,055\end{array}$ & $\begin{array}{c}- \\
0,078\end{array}$ & $\begin{array}{c}- \\
0,136 \\
\end{array}$ & 0,494 & 1 & \\
\hline NDGC & 0,111 & 0,212 & 0,122 & $\begin{array}{c}- \\
0,127\end{array}$ & $\begin{array}{c}- \\
0,133\end{array}$ & 0,002 & $\begin{array}{c}- \\
0,079\end{array}$ & 0,044 & $\begin{array}{c}- \\
0,202\end{array}$ & 0,366 & 0,236 & 1 \\
\hline
\end{tabular}

Os números representam o coeficiente de correlação pairwise entre as variáveis. T1, T2, T3 e TM são as variáveis de transparência de acordo com o trabalho de Leuz et al. (2003). Beta (B), Custo médio ponderado de capital no tempo corrente (WACC) e em $t+1$ (WACC $t+1$ ) são proxies do risco das empresas. Volatilidade (VOL), Índice book-to-market (BTM), Valor de Mercado (VM), Liquidez em bolsa (LB) e Adesão aos níveis diferenciados de governança corporativa da BM\&FBovespa (NDGC) são as variáveis de controles adotadas nos modelos. Os dados relativos ao fechamento fiscal do período de 2003 a 2012, para o WACC $\mathrm{t}+1$ os dados são referentes ao $2^{\circ}$ trimestre dos anos de 2004 a 2013. 
No que tange aos modelos econométricos estimados, a tabela 3 apresenta o resumo dos principais resultados dessa pesquisa, evidenciando as principais relações investigadas.

Tabela 3: Resumo dos coeficientes da regressão

\begin{tabular}{|c|c|c|c|}
\hline Variável & B & WACC & WACC t+1 \\
\hline T1 & $0,0405^{*}$ & 0,0181 & 0,0768 \\
\hline & $1,17 / 0,07$ & $0,23 / 0,82$ & $0,57 / 0,57$ \\
\hline T2 & 0,0409 & 0,0236 & 1,1383 \\
\hline T3 & $0,48 / 0,63$ & $0,04 / 0,97$ & $0,87 / 0,38$ \\
\hline TM & $-0,0293$ & $-0,5258^{*}$ & 0,0004 \\
\hline \multicolumn{2}{|l|}{$-0,52 / 0,60$} & $-1,86 / 0,06$ & $-0,2001$ \\
\hline & $0,1277^{\star *}$ & 0,0788 & $-0,19 / 0,85$ \\
\hline & $2,13 / 0,03$ & $0,36 / 0,72$ & Coeficiente \\
\hline
\end{tabular}

T1, T2, T3 e TM são as variáveis de transparência de acordo com o trabalho de Leuz et al. (2003). Beta (B), Custo médio ponderado de capital no tempo corrente (WACC) e em $\mathrm{t}+1$ (WACC $\mathrm{t}+1$ ) são proxies do risco das empresas. Os números representam os coeficientes estimados através das regressões de dados em painéis. ${ }^{*},{ }^{* *} \mathrm{e}^{* * *}$ correspondem à significância estatística nos níveis de $10 \%, 5 \%$ e $1 \%$, respectivamente. Os erros-padrão computados para todas as regressões são robustos a heterocedasticidade e a autocorrelação.

Nas regressões cuja variável dependente foi o beta $(B)$, somente os modelos com T1 e TM apresentaram resultados estatisticamente significantes no nível de 10\% e 5\% respectivamente. Contudo, ao contrário do que apontam os estudos de Skaife et al. (2004) e Lameira (2007) nos quais foram encontradas relações negativas da governança corporativa com o beta, nesse estudo a relação encontrada entre o beta e as variáveis T1 e TM apresenta sentido positivo, ou seja, quanto melhor o nível de governança corporativa maior o risco sistemático das empresas. Dessa forma, esse resultado corrobora o resultado da pesquisa de Salmasi (2007) que evidenciou uma relação positiva e significativa entre o beta e a governança corporativa. Para Salmasi (2007) essa relação poderia ser explicada pelo fato de que empresas que apresentam maior risco talvez busquem melhores práticas de governança corporativa com o intuito de mitigar, frente aos investidores, esses riscos.

Analisando o Painel A da tabela 4, observa-se, de acordo com o esperado, uma relação positiva e significante no nível de $1 \%$ entre a volatilidade (VOL) e o beta em todas as regressões.

Tabela 4: Coeficientes dos modelos de regressão

\begin{tabular}{|c|c|c|c|c|}
\hline \multirow[b]{2}{*}{ T1 } & \multicolumn{4}{|c|}{ Beta } \\
\hline & $0,0405^{*}$ & & & \\
\hline T2 & & 0,0409 & & \\
\hline T3 & & & $-0,0293$ & \\
\hline TM & & & & $0,1277^{* *}$ \\
\hline NDGC - Nível 1 & 0,0296 & 0,0102 & $-0,0053$ & $-0,0059$ \\
\hline NDGC - Nível 2 & $-0,0201$ & $-0,0652^{* *}$ & $-0,0449$ & $-0,0372$ \\
\hline NDGC - Novo Mercado & 0,0187 & $-0,005$ & 0,0183 & 0,0152 \\
\hline VOL & $0,0435^{\star \star \star}$ & $0,0483^{\star \star \star}$ & $0,0396^{* * *}$ & $0,0389^{* \star *}$ \\
\hline BTM & $-0,0091$ & $-0,0092$ & $-0,0038$ & $-0,004$ \\
\hline VM & 0,0074 & 0,0063 & 0,0199 & 0,0214 \\
\hline LB & 0,0498 & 0,059 & 0,0294 & 0,0301 \\
\hline Intercepto & $0,4065^{* *}$ & $0,4690^{* *}$ & 0,2557 & 0,2308 \\
\hline Controlado por Ano & Sim & Sim & Sim & Sim \\
\hline Controlado por Setor & Sim & Sim & Sim & Sim \\
\hline
\end{tabular}




\begin{tabular}{|c|c|c|c|c|}
\hline \multicolumn{5}{|c|}{ Painel B: Modelo com WACC como variável dependente } \\
\hline & \multicolumn{4}{|c|}{ WACC } \\
\hline T1 & 0,0181 & & & \\
\hline T2 & & 0,0236 & & \\
\hline T3 & & & $-0,5258^{*}$ & \\
\hline TM & & & & 0,0788 \\
\hline NDGC - Nível 1 & $-0,15$ & $-0,1461$ & $-0,2115$ & $-0,2144$ \\
\hline NDGC - Nível 2 & 0,2872 & 0,2395 & 0,2377 & 0,2407 \\
\hline NDGC - Novo Mercado & $0,6840^{* * *}$ & $0,6759^{\star * *}$ & $0,5951^{* * *}$ & $0,5908^{* * *}$ \\
\hline VOL & 0,0089 & 0,0084 & 0,0267 & 0,0252 \\
\hline BTM & $-0,5778^{* * *}$ & $-0,5577^{* * *}$ & $-0,5380^{* * *}$ & $-0,5373^{* * *}$ \\
\hline VM & $-0,0038$ & 0,0046 & 0,0955 & 0,0969 \\
\hline LB & 0,0935 & 0,0897 & $-0,0135$ & $-0,0138$ \\
\hline B & 0,0956 & 0,0955 & 0,0445 & 0,0391 \\
\hline Intercepto & $-1,6869$ & $-1,8262$ & $-3,0302^{* * *}$ & $-3,0415^{\star * *}$ \\
\hline Controlado por Ano & Sim & Sim & Sim & Sim \\
\hline Controlado por Setor & Sim & Sim & Sim & Sim \\
\hline \multicolumn{5}{|c|}{ Painel C: Modelo com WACC $\mathrm{t}+1$ como variável dependente } \\
\hline & \multicolumn{4}{|c|}{ WACC $t+1$} \\
\hline T1 & 0,0768 & & & \\
\hline T2 & & 1,1383 & & \\
\hline T3 & & & 0,0004 & \\
\hline TM & & & & $-0,2001$ \\
\hline NDGC - Nível 1 & $-0,0731$ & $-0,5479^{* *}$ & $-0,5064$ & $-0,5065$ \\
\hline NDGC - Nível 2 & 0,2898 & 0,3784 & $-0,0174$ & $-0,0181$ \\
\hline NDGC - Novo Mercado & $0,8059^{\star * *}$ & 0,4323 & $-0,2872$ & $-0,2873$ \\
\hline VOL & $0,1281^{* *}$ & 0,103 & 0,0877 & 0,0883 \\
\hline BTM & $-0,7864^{\star * *}$ & $-0,4859^{* *}$ & $-0,0334$ & $-0,0336$ \\
\hline VM & $-0,0536$ & 0,143 & $0,4048^{*}$ & $0,4052^{*}$ \\
\hline LB & 0,1252 & $-0,0895$ & $-0,4277$ & $-0,4284$ \\
\hline B & 0,4845 & $1,9266^{\star *}$ & 0,8425 & 0,8399 \\
\hline Intercepto & $-2,5075^{\star}$ & $-6,2464^{* * *}$ & $-8,8605^{* *}$ & $-8,8177^{\star *}$ \\
\hline Controlado por Ano & Sim & Sim & Sim & Sim \\
\hline Controlado por Setor & Sim & Sim & Sim & Sim \\
\hline
\end{tabular}

Para o segundo grupo de regressões, que tem como variável dependente o Custo Médio Ponderado de Capital (WACC) no período corrente, somente o modelo com T3 apresentou resultado significante no nível de 10\%, como pode ser observado na Tabela 3 . Ao contrário do beta, no modelo contendo as variáveis WACC e T3 a relação encontrada foi negativa, obtendo-se, assim, um resultado de acordo com o esperado e indicado nas pesquisas de Skaife et al. (2004) e Lameira (2007) - apesar de nesses estudos ter sido utilizado somente o WACC em t+1. Isso indica que quanto menos os resultados são gerenciados, maior é o nível de governança corporativa da empresa e menor é o seu custo médio ponderado de capital e, por conseguinte, menor é o risco interno da estrutura de capital da firma. Analisando conjuntamente os resultados encontrados nas regressões de beta e WACC e tendo em vista que o beta além de proxy de risco sistemático pode ser considerado como uma proxy do custo do capital próprio, conforme Salmasi (2007), infere-se que na amostra estudada melhores níveis de governança corporativa aumentam o custo do capital próprio, mas em contrapartida, reduzem o custo de capital de terceiros de tal forma que o custo médio ponderado de capital seja também reduzido.

No painel B da Tabela 4 pode-se observar que a variável book-to-market (BTM) possui relação significante com o WACC ao nível de 1\% e com sentido. Isso indica que quanto menos estiver valorizada a ação da empresa em comparação com o seu patrimônio menor será o seu risco 
medido através do WACC. Destaca-se ainda que a adesão ao Novo Mercado apresenta relação positiva e significante ao nível de $1 \%$ com o WACC. Ainda que seja outra proxy para o risco, esse resultado também corrobora o encontrado por Salmasi (2007).

Por fim, os modelos que utilizam o WACC em t+1 não apresentaram nenhuma relação significante com a transparência, conforme reportado no painel B da Tabela 4.

\section{CONCLUSÕES}

Esse estudo teve como principal objetivo verificar a relação existente entre o nível de transparência e o risco das empresas não financeiras negociadas na BM\&FBovespa no período de 2003 a 2012. Para isso, as variáveis de transparências propostas por Leuz et al. (2003) foram regredidas contra as proxies de risco: beta do CAPM (B), Custo Médio Ponderado de Capital (WACC) no tempo corrente e Custo Médio Ponderado de Capital em t+1 (WACC t+1). Para controlar os modelos propostos foram utilizadas as variáveis de volatilidade das ações (VOL), o próprio beta, o índice book-to-market (BTM), o valor de mercado (VM), a liquidez em bolsa (LB), a adesão aos níveis diferenciados de governança corporativa da BM\&FBovespa (NDGC) e dummies para Ano e Setor.

Utilizando regressões em painéis desbalanceados com Efeitos Aleatórios, exceto para o modelo com WACC em t+1 e TM, com erros-padrão robustos à heterocedasticidade e à autocorrelação, verificou-se uma relação positiva e significante ao nível de $10 \%$ e $5 \%$ entre o beta (B) e as variáveis T1 e TM respectivamente. Apesar desse resultado contrariar os estudos de Skaife et al. (2004) e de Lameira (2007), ele corrobora com a pesquisa de Salmasi (2007), que também encontrou uma relação positiva entre o nível de governança corporativa - medido pelo NDGC - e o beta do CAPM (que nesse caso foi utilizado como proxy do custo de capital próprio).

Por outro lado, considerando o WACC no tempo corrente como variável dependente, essa pesquisa obteve uma relação negativa e significante ao nível de 10\% tendo T3 como regressor. Esse resultado se alinha com as hipóteses inicias e coaduna com as pesquisas de Skaife et al. (2004) e de Lameira (2007) que encontraram uma relação inversa entre qualidade da governança corporativa e risco da estrutura de capital da firma. Por sua vez, para as regressões de transparência com o WACC $t+1$, esse trabalho não encontrou nenhuma relação significante com transparência ao nível de pelo menos $10 \%$.

Dessa forma, essa pesquisa encontrou resultados distintos na relação entre transparência e risco dependendo da proxy de risco utilizada, obtendo uma relação positiva (T1 e TM contra beta) ou negativa (T3 contra WACC), em ambos os casos significantes pelo menos ao nível de $10 \%$. Essas constatações evidenciam a importância da modelagem econométrica no processo de entendimento das relações entre as variáveis, principalmente no que tange à correta avaliação das variáveis e escolha das proxies mais precisas para os indicadores financeiro-econômicos. Ademais, infere-se que nessa amostra uma maior qualidade da governança corporativa aumenta o custo do 
capital próprio, mas, em contrapartida, reduz o custo de capital de terceiros de tal forma que o custo médio ponderado de capital da firma seja também reduzido.

Com essa pesquisa também pode-se inferir que para mercados de capital menos desenvolvidos, como o mercado brasileiro, a relação entre o risco sistemático das ações e a governança corporativa pode ser menos influenciada pelos atributos corporativos observáveis (indicadores financeiros) do que o esperado pela literatura. Inferência essa que também é reforçada pelas relações positivas e significantes encontradas tanto entre a Adesão aos Níveis Diferenciados de Governança Corporativa da BM\&FBovespa (NDGC) e o beta - encontradas nesse estudo e em Salmasi (2007) - quanto com o WACC no tempo corrente - encontradas nesse trabalho.

Como sugestões de trabalhos futuros propõem se verificar a qualidade do disclosure de empresas que enfrentam dificuldades financeiras.

\section{REFERÊNCIAS}

BARROS, L. A. B. C.; FAMÁ, R.; SILVEIRA, H. P.. Aspectos da teoria de portfólio em mercados emergentes: uma análise de aproximações para a taxa livre de risco no Brasil. In: SEMEAD - SEMINÁRIO DE ADMINISTRAÇÃO - FEA-USP, 6. Anais. São Paulo, 2003.

BAUM, C. F.. Residual diagnostics for cross-section time series regression models. Stata Journal, v.1, n.1, p.101-104, 2001.

BERLE, A. A.; MEANS, G. C.. The modern corporation and private property. New York: Macmillan, 1932. BREUSCH, T. S.; PAGAN, A. R.. The Lagrange multiplier test and its applications to model specification in econometrics. The Review of Economic Studies, v.47, n.1, p.239-253, 1980.

BUSCHMAN, R.; SMITH, A.. Transparency, financial accounting information, and corporate governance. Economic Policy Review, v. 9, n. 1, 2003.

BUENO, A. F.. Problemas de disclosure no Brasil: o caso das empresas com ações no exterior. Caderno de Estudos, São Paulo, n.20, p.1-13, 1999.

CASTRO, F. H. F.; CONCEIÇÃO, P. M.; SANTOS, D. A.. A relação entre o nível voluntário de transparência e o custo de capital próprio das empresas brasileiras não-financeiras. Revista Eletrônica de

Administração, Porto Alegre, v.17, n.3, p.617-635, 2011.

CORREIA, L. F.. Um índice de governança para empresas no Brasil. Tese (Doutorado) - Universidade Federal de Minas Gerais, Belo Horizonte, 2008.

DAMODARAN, A.. Avaliação de investimentos: ferramentas e técnicas para a determinação do valor de qualquer ativo. 4 ed. Rio de Janeiro: Qualitymark, 2002.

FERNANDES, N. G. G.; FERREIRA, M. A.. The evolution of earnings management and firm valuation: a cross-country analysis. Ljubljana Meetings Paper, 2007.

FERNANDES, N. G. G.; FERREIRA, M. A.. Does international cross-listing improve the information environment. Journal Of Financial Economics, v.88, n.2, p.216-244, 2008.

GREENE, W.. Econometric Analysis. 5 ed. Upper Saddle River: Prentice Hall, 2008.

HAUSMAN, J. A.. Specification tests in econometrics. Journal of the Econometric Society, v.46, n.6, p.1251-1271, 1978. 
HOECHLE, D.. Robust standard errors for panel regressions with cross-sectional dependence. Stata Journal, v.7, n.3, p.281-311, 2007.

LA PORTA, R.. Investor protection and corporate governance. Journal Of Financial Economics, v.58, n.12, p.3-27, 2000.

LAMEIRA, V. J.. Governança corporativa, risco e desempenho das companhias abertas brasileiras: uma análise do relacionamento entre as práticas de governança corporativa, o risco e o desempenho das companhias abertas brasileiras. Tese (Doutorado em Administração) - Pontifícia Universidade Católica do Rio de Janeiro, Rio de Janeiro, 2007.

LANZANA, A. P.. Relação entre disclosure e governança corporativa das empresas brasileira. Dissertação (Mestrado em Administração) - Universidade de São Paulo, São Paulo, 2004.

LANZANA, A. P.; SILVEIRA, A. D. M.; FAMÁ, R.. Existe relação entre disclosure e governança corporativa no Brasil?. In: ENANPAD, 30. Anais. Salvador, 2006.

LEUZ, C.; NANDA, D.; WYSOCKI, P. D. Earnings management and investor protection: an international comparison. Journal Of Financial Economics, v.69, n.3, p.505-527, 2003.

LIU, C.; UCHIDA, K.; YANG, Y.. Corporate governance and firm value during the global financial crisis: evidence from China. International Review of Financial Analysis, v.21, p.70-80, 2012.

LOPES, A. B.; WALKER, M.. Firm-level incentives and the informativeness of accounting reports: an experiment in Brazil. Working Papers Series, 2008.

MALACRIDA, M. J. C.; YAMAMOTO, M. M.. Governança corporativa: nível de evidenciação das informações e sua relação com a volatilidade das ações do IBOVESPA. Revista de Contabilidade e Finanças da USP, São Paulo, p.65-79, 2006.

MARTINEZ, A. L.. Gerenciamento de resultados no Brasil: um survey da literatura. Brazilian Business Review, v.10, n.4, p.1-31, 2013.

MOKHTAR, E. S.; MELLETT, H.. Competition, corporate governance, ownership structure and risk reporting. Managerial Auditing Journal, v.28, n.9, p.838-865, 2013.

PEIXOTO, F. M.. Governança corporativa, desempenho, valor e risco: estudo das mudanças em momentos de crise. Tese (Doutorado em Administração) - Universidade Federal de Minas Gerais, Belo Horizonte, 2012.

ROGERS, P.; SECURATO, J. R.. Estudo comparativo no mercado brasileiro do capital asset pricing model (CAPM): modelo 3-fatores de Fama e French e reward beta approach. RAC-Eletrônica, v.3, n.1, p.159-179, 2009 .

SALMASI, S. V.. Governança corporativa e custo de capital próprio no Brasil. Dissertação (Mestrado em Administração) - Universidade de São Paulo, São Paulo, 2007.

SILVEIRA, A. D.. Governança Corporativa e Estrutura de Propriedade: determinantes e relação com o desempenho das empresas no Brasil. Tese (Doutorado em Administração) - Universidade de São Paulo, São Paulo, 2004.

SILVEIRA, A. M.; BARROS, L. A. B. C.. Determinantes da qualidade da governança corporativa das companhias abertas brasileiras. Revista Eletrônica de Administração, Porto Alegre, v.14, n.61, p.512-540, 2008.

SKAIFE, H. A; COLLINS, D. W.; LAFOND, R.. Corporate governance and the cost of equity capital. Working Papers Series, 2004.

SKINNER, D. J. The investment opportunity set and accounting procedure choice: preliminary evidence. Journal of Accounting And Economics, v.16, n.4, p.407-445, 1993.

SROUR, G.. Práticas diferenciadas de governança corporativa: um estudo sobre a conduta e a performance das firmas brasileiras. Revista Brasileira de Economia, Rio de Janeiro, p.635-674, 2005. 
WOOLDRIDGE, J. M.. Econometric analysis of cross section and panel data. London: MIT Press, 2002.

WONG, S.. Uses and limits of conventional corporate governance instruments: analysis and guidance for reform. Integrated Version Northwestern Law \& Econ Research Paper, 2009. 